\title{
Small-scale mixing, large-scale advection and stratospheric tracer distributions
}

\author{
J. Vanneste* \\ School of Mathematics, University of Edinburgh, Edinburgh, UK \\ May 31, 2004
}

${ }^{*}$ Corresponding author address: J. Vanneste, School of Mathematics, University of Edinburgh, King's Buildings, Mayfield Road, Edinburgh EH9 3JZ, United Kingdom. Email: J.Vanneste@ed.ac.uk 
The vertical mixing of tracers in the stratosphere is mainly due to patches of three-dimensional turbulence which are highly intermittent in time and space. A simple heuristic model of this form of mixing is developed and employed to examine the effect of small-scale mixing on passive stratospheric tracers. The model is based on random-walk ideas, and it leads to an analogue of the usual advection-diffusion equation in which the diffusion operator is replaced by a convolution operator taking the intermittency of the mixing into account. In its simplest form, this operator is defined by two parameters; these are estimated from mid-latitude lower-stratospheric balloon data using a stochastic model of turbulent patches.

The behaviour of tracer distributions in some idealised flows shows how intermittency makes mixing less effective in damping the small-scale tracer fluctuations which arise through differential advection. This has consequences for stratospheric tracer distributions which are demonstrated using numerical simulations based on observed stratospheric winds. Specifically, the new model of mixing leads to a horizontal tracer spectrum which is shallower, and closer to a $k^{-2}$ power law, than the spectrum obtained with a diffusive parameterization of mixing. The horizontal scale below which intermittent mixing differs significantly from diffusion is estimated to be $15 \mathrm{~km}$ or so; remarkably, this coincides with the dissipative scale below which dissipation by small-scale mixing is crucial for tracer evolution. 


\section{Introduction}

Models of stratospheric tracer transport and mixing generally use the advection-diffusion equation as a starting point. This is partly justified by the nature of the stratospheric flow which can usefully be separated into a large-scale component, dominated by layerwise two-dimensional motion, and a small-scale component, dominated by three-dimensional turbulence (which results from gravity-wave breaking and shear instabilities). Thus, while the large-scale advection is described explicitly, the small-scale mixing associated with threedimensional turbulence is parameterized by diffusion. This diffusive parameterization is widely accepted (although the value of the corresponding diffusivity remains controversial; see e.g. Alisse et al., 2000; Joseph et al., 2003), but, in fact, its adequacy is questionable. The main reason for this uncertainty is the rather special form that small-scale mixing takes in the stratosphere: as a result of the strong stratification and of the heterogeneity in the turbulent-energy input, three-dimensional turbulence is confined within patches that are localized in space and time, and occupy only a small volume fraction of the atmosphere (e.g. Dewan, 1981; Alisse \& Sidi, 2000). The consequent intermittency in the small-scale mixing may, one suspects, lead to tracer evolution that cannot be modelled accurately by diffusion. The present paper examines the extent to which this is the case by developing a parameterization of small-scale mixing that takes the intermittency of the mixing into account. Comparison between tracer distributions obtained with this novel parameterization of mixing and with diffusion, in idealized and in realistic flows, indicate significant non-diffusive effects.

Our parameterization of small-scale mixing is based on a heuristic model originally due to Dewan $(1980,1981)$ and recently revisited by Vanneste \& Haynes (2000). In this model, 
best described in Lagrangian terms, fluid particles are assumed to encounter a succession of turbulent patches at random times and, as a result, undergo a succession of random displacements. Only the (irreversible) cross-isentropic displacements (loosely referred to as vertical) are in fact taken into account, since they dominate the dissipative process (Haynes \& Anglade, 1997). Vanneste \& Haynes (2000) used the theory of continuous-time random walks (Montroll \& Weiss, 1965) to describe the vertical dispersion of fluid particles predicted by this model. We extend their results by considering the combined effect of mixing and large-scale advection.

With an assumption of statistical independence between successive encounters of fluid particles with turbulent patches, we derive an evolution equation for the concentration of a passive tracer that is advected by a large-scale velocity field, and mixed vertically by turbulent patches (section 2). In this so-called master equation, the effect of the small-scale mixing is represented by a relatively simple convolution operator which replaces the usual second derivative representing diffusion. The definition of this operator requires knowledge of the probability density function (pdf) of the vertical displacements that result when a fluid particle encounters a turbulent patch; for simplicity (and because it is poorly constrained by observations), we choose this pdf to be either Gaussian or exponential, and we examine the differences between these two choices. With either choice, only two parameters need to be determined: the variance of the vertical displacements, and the average waiting time, that is, the average time between encounters of a fluid particle with turbulent patches. We devote Appendix A to a discussion of the relationship between these two parameters and more directly observable features of the turbulent patches. This Appendix draws on results of Alisse et al. (2000) to infer realistic values for the parameters from the balloon data of Alisse \& Sidi (2000). 
The operator parameterizing small-scale mixing in the master equation reduces to standard diffusion when acting on large-scale tracer fluctuations. Its behaviour is markedly different, however, for fluctuations with small vertical scales (of the order of the typical vertical displacement associated with each encounter with turbulent patches): as the tracer scale tends to zero, the damping rate becomes scale independent, given by the inverse of the average waiting time. This effect is crucial for flows, such as the stratospheric flow, with both horizontal strain and vertical shear, since the formation of tracer structures with very small vertical scales is then inevitable (Haynes \& Anglade, 1997). In section 3, we examine this point in some details by studying the evolution of passive tracers in simple strained flows. A clear conclusion is that the intermittency of the mixing reduces its efficiency at damping small-scale tracer fluctuations and leads, for instance, to filamentary structures with sharper edges than those obtained for diffusive mixing. We estimate the horizontal scale at which this effect appears in the stratosphere to be of the order of $15 \mathrm{~km}$; interestingly, this is also the scale at which dissipative effects (parameterized by diffusion) are thought to become important (Haynes \& Anglade, 1997; Sparling \& Bacmeister, 2001).

The impact of the intermittency of the small-scale mixing on stratospheric tracer distribution is examined in a more realistic setting in section 4 . There, we compute the horizontal power spectrum for a passive tracer that is forced at large scale, advected by realistic (winter) stratospheric winds, and dissipated at small scale according to our parameterization of smallscale mixing. This spectrum is calculated over a wide range of scales using the WKB method developed by Antonsen et al. (1996) and applied to the stratospheric context by Haynes \& Vanneste (2004). We follow closely Haynes \& Vanneste (2004) in taking into account both the horizontal strain and vertical shear of the stratospheric winds, but we replace the diffusion they employed by our parameterization of intermittent mixing. Compared with that 
obtained with diffusion, the intermittent-mixing spectrum is found to be shallower at small scales, say from $20 \mathrm{~km}$ down, and, for realistic values of the parameters, well approximated

by a $k^{-2}$ power law. This result is related to spectra inferred from aircraft measurements of $\mathrm{N}_{2} \mathrm{O}$ and $\mathrm{O}_{3}$ by Bacmeister et al. (1996) and Sparling \& Bacmeister (2001).

\section{Model of small-scale mixing}

\section{$2.1 \quad$ Random-walk model}

Following Dewan $(1980,1981)$ and Vanneste \& Haynes (2000), we assume that mixing in the stratosphere is concentrated within randomly distributed turbulent patches. Taking a Lagrangian viewpoint, we consider the motion of a single fluid particle, and we model its vertical (or more accurately cross-isentropic) displacements due to mixing by a continuoustime random walk (Montroll \& Weiss, 1965; Weiss \& Rubin, 1983); that is, we model the particle motion by a succession of random vertical steps, each describing the net effect of an encounter with a turbulent patch, separated by random time intervals during which the motion is purely horizontal. Such a stochastic model is completely defined by the pdf $\Phi(l, \tau)$ for the size $l$ of the vertical steps and for the waiting times $\tau$, i.e. the time intervals between encounters with turbulent patches. See Figure 1 for an illustration.

For simplicity, a separable form for $\Phi(l, \tau)$ is assumed: $\Phi(l, \tau)=p(l) \psi(\tau)$. In what follows we make the further assumption that the waiting times obey a Poisson distribution:

$$
\psi(\tau)=\alpha \exp (-\alpha \tau)
$$

This particular case of continuous-time random walk is termed randomized random walk (Weiss \& Rubin, 1983). Here, $\alpha^{-1}$ is the average waiting time; it is a crucial parameter 
which, when compared with other time scales relevant to the tracer evolution, can be used to estimate the importance of the intermittency of the small-scale mixing (Vanneste \& Haynes, 2000). Note that the Poisson distribution (2.1) assumes the independence of the successive turbulent events experienced by a fluid particle. Arguably this may be unrealistic since the finite time taken for turbulent patches to restratify is likely to induce some memory effect (Vanneste \& Haynes, 2000). However, in the absence of precise information about the form of $\psi(\tau)$ in the stratosphere, the Poisson form provides a straightforward first approximation.

The displacement pdf $p(l)$ is related to the statistical properties of turbulent patches in a somewhat involved way. In the absence of reliable estimate, we consider arbitrary forms of $p(l)$, with as only hypotheses the symmetry $p(-l)=p(l)$ and the finiteness of the displacement variance

$$
\sigma^{2}=\int_{-\infty}^{\infty} p(l) \mathrm{d} l
$$

Two possible forms of $p(l)$, both parameterized solely by $\sigma$, will be used for illustrative purposes: they are the Gaussian and exponential distributions,

$$
p_{\mathrm{g}}=\frac{1}{\sqrt{2 \pi} \sigma} \exp \left(-\frac{l^{2}}{2 \sigma^{2}}\right) \quad \text { and } \quad p_{\mathrm{e}}=\frac{1}{\sqrt{2} \sigma} \exp \left(-\frac{\sqrt{2}|l|}{\sigma}\right) .
$$

A comparison of the results obtained with each pdf allows a first assessment of the sensitivity of the model to the precise form of $p(l)$.

In Appendix A, we present in some details a model of mixing within turbulent patches which in principle allows the determination of $\alpha$ and $\sigma^{2}$ (or indeed of $p(l)$ ) from observed statistics of turbulent patches. This model has been used in Alisse et al. (2000) to derive an estimate of stratospheric diffusivity from balloon data. From our perspective, the main results of that paper are the estimates

$$
\alpha \approx 10^{-5} \mathrm{~s}^{-1} \text { and } \sigma \approx 50 \mathrm{~m}
$$


also discussed in Appendix A. With these values and with the assumed pdfs (2.1) and (2.2), the random-walk model of small-scale mixing is entirely determined.

An advantage of the model, in addition to its plausibility as a heuristic model of mixing, is that a simple evolution equation can be derived for the concentration of a passive tracer which is both advected by large-scale horizontal winds and mixed vertically according to the random-walk prescription. We derive this evolution equation in the next section.

\subsection{Master equation}

The position $\mathbf{x}(t)$ of a fluid particle which is advected by a (divergenceless) velocity field $\mathbf{v}(\mathbf{x}, t)$ and undergoes a randomized random walk in the vertical satisfies the equation

$$
\dot{\mathbf{x}}=\mathbf{v}(\mathbf{x}, t)+\eta(t) \hat{\mathbf{z}} .
$$

Here, $\hat{\mathbf{z}}$ is a vertical unit vector and $\eta(t)$ is a white Poisson process, formally defined by

$$
\eta(t)=\sum_{j=1}^{n(t)} l_{j} \delta\left(t-t_{j}\right),
$$

where $n(t)$ is a Poisson counting process, and the $t_{j}$ are distributed uniformly with density $\alpha$ in $(0, t)$ (see, e.g., Hanggi, 1985). Each time $t_{j}$ corresponds to an encounter of the fluid particle with a turbulent patch, resulting in the net random displacement $l_{j}$ with pdf $p(l)$.

Corresponding to the stochastic differential equation (2.4), one can derive a master equation governing the evolution of the particle-position pdf $P(\mathbf{x}, t)$. This pdf can be interpreted as the concentration of a passive tracer averaged over realizations of the random process in (2.4) or, in other words, averaged over an ensemble of turbulent patches. Thus, the master equation is the desired evolution equation for the tracer concentration.

A detailed derivation of the master equation is given in Appendix B. It starts by consid- 
ering the functional expression

$$
P(\mathbf{x}, t)=\langle\delta(\mathbf{x}-\mathbf{x}(t))\rangle,
$$

where $\langle\cdot\rangle$ denotes average over realizations and $\mathbf{x}(t)$ is a solution of (2.4). Using (2.4) to evaluate the time derivative of the right-hand side leads to

$$
\partial_{t} P(\mathbf{x}, t)+\nabla \cdot[\mathbf{v} P(\mathbf{x}, t)]=\alpha \int_{-\infty}^{\infty} p(l)[P(\mathbf{x}-l \hat{\mathbf{z}}, t)-P(\mathbf{x}, t)] \mathrm{d} l .
$$

Here, the convolution term is associated with the randomized random walk and can thus be regarded as a parameterization of the mixing associated with intermittent patches of turbulence. This term is exactly the one found for a randomized random walk without advection (Montroll \& Weiss, 1965; Weiss \& Rubin, 1983; Vanneste \& Haynes, 2000); in other words, advection and mixing are represented by two separate terms in the master equation. This is likely a particularity of the Poisson process: when the noise process is not Poisson, i.e. when there is dependence between successive turbulent events due to a memory effect, advection and mixing probably cannot be separated in such a straightforward manner. A convenient reformulation of (2.6) uses the Fourier transform of $p(l)$ defined as

$$
\hat{p}(m)=\int_{-\infty}^{\infty} \mathrm{e}^{-\mathrm{i} l m} p(l) \mathrm{d} l .
$$

Formally, (2.6) can be written as

$$
\partial_{t} P(\mathbf{x}, t)+\nabla \cdot[\mathbf{v} P(\mathbf{x}, t)]=-d\left(-\mathrm{i} \partial_{z}\right) P(\mathbf{x}, t),
$$

where the operator $d\left(-\mathrm{i} \partial_{z}\right)$ is defined by the corresponding function

$$
d(m)=\alpha[1-\hat{p}(m)]
$$

In this form, the master equation (2.7) is seen to be a generalization of the usual advectiondiffusion equation in which the diffusion operator $\kappa \partial_{z z}^{2}$ is replaced by the more general 
operator $-d\left(-\mathrm{i} \partial_{z}\right)$. In general, $d(m)$ is not a polynomial in $m$, hence the operator $d\left(-\mathrm{i} \partial_{z}\right)$ is a non-local (or pseudodifferential) operator.

\subsection{Interpretation}

The effect of the new representation of small-scale mixing is best understood by considering the evolution of tracer concentration in one, vertical, dimension and in the absence of advection. The Fourier transform of $(2.7)$ with $\mathbf{v}(\mathbf{x}, t)=0$ is simply

$$
\partial_{t} \hat{P}(m, t)=-d(m) \hat{P}(m, t)
$$

where $m$ is the vertical wavenumber. The general solution, found to be

$$
\hat{P}(m, t)=\exp [-d(m) t] \hat{P}(m, 0),
$$

describes the damping of tracer concentration fluctuations that results from mixing: the fluctuations are seen to decay exponentially, with decay rate $d(m)$. This provides a direct interpretation for the function $d(m)$ defined in $(2.8)$.

When the mixing is diffusive, the damping rate is quadratic in the wavenumber, $d(m)=$ $\kappa m^{2}$. This simple form is recovered as a limiting case of more general $d(m)$, since a McLaurin expansion of $\hat{d}(m)$ yields

$$
d(m) \sim \alpha \sigma^{2} m^{2} / 2 \text { for } m \ll \sigma^{-1} \text {. }
$$

This shows that diffusion with diffusivity

$$
\kappa=\alpha \sigma^{2} / 2
$$

provides a good parameterization of mixing for vertical scales much larger than $\sigma$, the typical vertical displacement caused by turbulent patches. On smaller, $O(\sigma)$, scales, however, the 
intermittency in the mixing results in a damping of tracer fluctuations that differs significantly from that predicted by diffusion. In particular, for $m \gg \sigma^{-1}$, realistic functions $\hat{p}(m)$ tend to zero, leading to

$$
d(m) \sim \alpha \text { for } m \gg \sigma^{-1}
$$

This results indicates that the decay rate of small-scale tracer fluctuations is given by $\alpha$, the inverse of the average waiting, and is thus scale independent. This behaviour is markedly different from that of diffusion, of course, which has $d(m) \rightarrow \infty$ for $m \rightarrow \infty$; it can be interpreted as a reduction of the efficiency of the mixing that results from its intermittency, and it will be seen to have important consequences for tracer distributions in realistic settings. Note that the diffusivity given by (2.9) allows a meaningful comparison between the randomwalk and diffusive models of small-scale mixing; this will be used throughout the paper.

Figure 2 compares the decay rate $d(m)$ associated with diffusion with those associated with intermittent mixing for the two step pdfs $p(l)$ in $(2.2)$. These damping rates are given by

$$
d_{\mathrm{g}}(m)=\alpha\left[1-\exp \left(-\sigma^{2} m^{2} / 2\right)\right] \quad \text { and } \quad d_{\mathrm{e}}(m)=\frac{\alpha \sigma^{2} m^{2}}{2+\sigma^{2} m^{2}}
$$

Clearly, the decay rate is overestimated by the diffusive approximation, with a diverging, and arguably non-physical, behaviour for large $m$. The Gaussian step distribution leads to a slightly larger decay rate than the exponential distribution, although both tend to the same value, $\alpha$, for large $m$.

\section{Simple models of tracer evolution}

In this section, we examine the coupling between intermittent mixing and large-scale advection using idealised models. The emphasis is on comparing results obtained using the 
random-walk model (2.7) with standard results obtained using diffusion.

\subsection{Vertically strained flow}

In the atmospheric context, tracer spatial scales are continuously reduced by differential advection. Therefore, in initial-value problems, the non-diffusive effects which appear at small scales necessarily play a role for large times. To illustrate this, we start by considering the interaction between intermittent mixing and a one-dimensional (vertical) strain flow $-\gamma z$. From (2.7) the evolution equation for the tracer concentration can be written

$$
\partial_{t} P(z, t)-\gamma \partial_{z}[z P(z, t)]=-d\left(-\mathrm{i} \partial_{z}\right) P(z, t)
$$

Because the velocity field is divergent, it is convenient to consider the mixing ratio $C(z, t)=$ $\exp (-\gamma t) P(z, t)$ which satisfies

$$
\partial_{t} C(z, t)-\gamma z \partial_{z} C(z, t)=-d\left(-\mathrm{i} \partial_{z}\right) C(z, t)
$$

Solutions to (3.1) can be sought in the form

$$
C(z, t)=A(t) \exp [\mathrm{im}(t) z]
$$

with time-dependent wavenumber $m(t)=m_{0} \exp (\gamma t)$. The amplitude $A(t)$ satisfies the equation

$$
\frac{\mathrm{d} A}{\mathrm{~d} t}=-d(m) A
$$

whose solution is

$$
A(t)=A_{0} \exp \left\{-\frac{1}{\gamma}\left[F\left(m_{0} \mathrm{e}^{\gamma t}\right)-F\left(m_{0}\right)\right]\right\},
$$

with

$$
F(m)=\int_{0}^{m} \frac{d\left(m^{\prime}\right)}{m^{\prime}} \mathrm{d} m^{\prime}
$$


The function $F(m)$ governs the decay of the tracer fluctuation amplitude. For diffusive mixing, it is simply given by

$$
F_{\text {diff }}(m)=\frac{\kappa m^{2}}{2}
$$

leading to the usual super-exponential decay of the amplitude. For intermittent mixing with Gaussian step pdf, it is given by

$$
F_{\mathrm{g}}(m)=\frac{\alpha}{2} \int_{0}^{\sigma^{2} m^{2} / 2} \frac{1-\mathrm{e}^{-x}}{x} \mathrm{~d} x=\frac{\alpha}{2}\left[\tilde{\gamma}+\ln \left(\frac{\sigma^{2} m^{2}}{2}\right)+E_{1}\left(\frac{\sigma^{2} m^{2}}{2}\right)\right],
$$

where $\tilde{\gamma}$ is the Euler constant and $E_{1}(\cdot)$ the exponential integral. For small $m$, the diffusive form of $F(m)$ is recovered. For large $m$, i.e. $m \gg \sigma^{-1}$, or, equivalently $t \gg-\gamma^{-1} \ln \left(\sigma m_{0}\right)$, $F(m) \sim(\alpha / 2) \ln \left(\sigma^{2} m^{2} / 2\right)$, leading to $A(t) \propto \exp (-\alpha t)$. Thus the long-time decay of tracer fluctuations is exponential, rather than super-exponential, with a decay rate $\alpha$. More generally, we can conclude that the intermittency results in a slowdown of tracer-fluctuation decay that is significant when $m(t) \sim \sigma^{-1}$. A similar behaviour is found for exponential step pdf. In this case $F(m)$ takes the simple form

$$
F_{\mathrm{e}}(m)=\frac{\alpha}{2} \ln \left(1+\frac{\sigma^{2} m^{2}}{2}\right)
$$

which has the same asymptotics as (3.3).

To illustrate the difference in behaviour between diffusion and intermittent mixing, we show in Figure 3 the dimensionless function $B(\gamma t)$ defined by

$$
A(t)=A_{0} \exp [\beta B(\gamma t)]
$$

and (3.2). Here,

$$
\beta=\frac{\alpha}{2 \gamma}
$$

is half the ratio of two time scales: the time scale of the strain $\gamma^{-1}$, and the average waiting time $\alpha^{-1}$. The initial vertical wavenumber has been taken as $m_{0}=10^{-2} \sigma^{-1}$ so that the non- 
diffusive effects are expected to become significant for $\gamma t \approx-\log \left(\sigma m_{0}\right)=\log 10^{2}=4.60 \cdots$. The Figure confirms this and demonstrates the much slower decay of the tracer amplitude $A(t)$ for intermittent mixing than for diffusion, with $B(\gamma t)$ asymptotically linear in the former case and exponential in the latter.

Another solution to (3.1) of interest is the stationary solution that obtains when the tracer mixing ratio is asymptotic to two different constants for $z \rightarrow \pm \infty$. The relevant equation, namely

$$
-\gamma z \partial_{z} C(z)=-d\left(-\mathrm{i} \partial_{z}\right) C(z)
$$

can be solved by Fourier transform. After some manipulations, one finds

$$
C(z)=c_{1} \int_{-\infty}^{\infty} \frac{\mathrm{e}^{\mathrm{i} m z-F(m) / \gamma}}{m} \mathrm{~d} m+c_{2}
$$

where $c_{1}$ and $c_{2}$ are arbitrary constants. In the diffusive case with $F_{\text {diff }}(m)=\kappa m^{2} / 2$, the integral can be evaluated by first considering its derivative with respect to $z$ then integrating the result; this leads to the expression

$$
C(z)=c_{1}^{\prime} \operatorname{erf}\left(\delta_{\operatorname{diff}} z\right)+c_{2}^{\prime}
$$

showing that the transition in the mixing ratio between its asymptotic values as $z \rightarrow \pm \infty$ takes place on the familiar inverse spatial scale

$$
\delta_{\text {diff }}=\sqrt{\gamma /(2 \kappa)}
$$

corresponding to a balance between strain and diffusion. In the non-diffusive case, a numerical calculation is required to obtain $C(z)$. An inverse scale similar to $\delta_{\text {diff }}$ can be introduced according to the expression

$$
\delta=\left|\frac{\sqrt{\pi} C^{\prime}(0)}{C(\infty)-C(-\infty)}\right|,
$$


which defines the non-dimensional slope of the tracer profile at $z=0$. A simple calculation provides $\delta$ in the form

$$
\delta=\frac{1}{2 \sqrt{\pi}} \int_{-\infty}^{\infty} \mathrm{e}^{-F(m) / \gamma} \mathrm{d} m .
$$

For an exponential step distribution, the integral on the right-hand side can be expressed in terms of $\Gamma$-functions as

$$
\delta_{\mathrm{e}}=\frac{\Gamma(\beta-1 / 2)}{\sqrt{2} \sigma \Gamma(\beta)}=\delta_{\mathrm{diff}} \frac{\sqrt{\beta} \Gamma(\beta-1 / 2)}{\Gamma(\beta)},
$$

where $\beta$ is defined in (3.6) and (3.4) has been used. The second equality shows that the slope $\delta_{\mathrm{e}}$ scaled by the diffusive estimate $\delta_{\text {diff }}$ depends only on $\beta$. The same holds true when the step pdf is Gaussian. In this case, the integral (3.8) defining $\delta_{\mathrm{g}}$ needs to be computed numerically.

Figure 4 displays $\delta_{\mathrm{g}} / \delta_{\text {diff }}$ and $\delta_{\mathrm{e}} / \delta_{\text {diff }}$ as functions of $\beta$. Both functions always exceed 1 , thus indicating that non-diffusive mixing leads to a sharper transition in tracer concentration than diffusive mixing. For long average waiting times, i.e. for small $\beta, \delta_{\mathrm{g}}$ and $\delta_{\mathrm{e}}$ are large and, in particular, become infinite for $\beta \leq 1 / 2$. This indicates that there is no steady profile for a strained tracer when $\alpha \leq \gamma$; thus, in an initial-value problem, the slopes $\delta_{\mathrm{g}}$ or $\delta_{\mathrm{e}}$ would grow without bounds, because the mixing is too inefficient to balance the strain. For short average waiting time, i.e. large $\beta$, the diffusive behaviour is of course recovered since $\delta_{\mathrm{g}}$ and $\delta_{\mathrm{e}}$ both tend to $\delta_{\text {diff }}$.

Figure 5 illustrates the difference between diffusive and intermittent mixing further by comparing the diffusive concentration profile (3.7) with that obtained using the randomwalk model with an exponential step distribution and $\beta=1$. For this particular value, the closed-form expression $C(z) \sim \operatorname{sgn} z[1-\exp (-\sqrt{2}|z| \sigma)]$ is readily derived. 


\subsection{Horizontally strained and vertical sheared flow}

The vertically strained flow of the previous section is not realistic; nevertheless, it illustrates the interplay between the reduction of scale that is associated with differential advection, and intermittent mixing. The reduction of vertical scale appears more realistically in the model considered by Haynes \& Anglade (1997) of a flow with horizontal strain and vertical shear.

The three-dimensional, incompressible velocity field in this model takes the form $(\gamma x,-\gamma y+$ $\lambda z, 0)$, where $\gamma$ is now the horizontal strain rate while $\lambda$ is the vertical shear. Neglecting the horizontal part of the mixing, we write the evolution equation for the tracer concentration as

$$
\partial_{t} P(\mathbf{x}, t)+\gamma x \partial_{x} P(\mathbf{x}, t)+(\lambda z-\gamma y) \partial_{y} P(\mathbf{x}, t)=-d\left(-\mathrm{i} \partial_{z}\right) P(\mathbf{x}, t) .
$$

A solution is found in the form

$$
P(\mathbf{x}, t)=A(t) \mathrm{e}^{\mathrm{i} \mathbf{k}(t) \cdot \mathbf{x}}
$$

with

$$
\mathbf{k}(t)=(k(t), l(t), m(t))=\left(k_{0} \mathrm{e}^{-\gamma t}, l_{0} \mathrm{e}^{\gamma t}, m_{0}-\lambda l_{0}\left(\mathrm{e}^{\gamma t}-1\right) / \gamma\right)
$$

With this result, the evolution equation for the amplitude $A(t)$, namely

$$
\frac{\mathrm{d} A(t)}{\mathrm{d} t}=-d[m(t)] A(t)
$$

is readily integrated. For simplicity, we assume that the initial vertical wavenumber satisfies $\gamma m_{0}=-\lambda l_{0}$, so that $m(t)=-\lambda l_{0} \exp (\gamma t)$. (This expression is also a good approximation to the exact solution for any $m_{0}$ for $\gamma t \gg 1$.) This allows the results of the previous section to be employed here: indeed, $m(t)$ evolves similarly in both cases, with the proviso that the initial value for $m_{0}$ is now $-\lambda l_{0}$. Thus, the conclusions drawn about the effect 
of intermittent mixing obtained with the vertically strained flow apply to the horizontally strained and vertically sheared flow as well. This is true, in particular, for the slowdown of tracer fluctuations associated with the intermittency of the mixing that appears when $m(t) \sim \sigma^{-1}$. Now, the interesting point is that the vertical and horizontal scales are related for $\gamma t \gg 1$. The condition $m(t) \sim \sigma^{-1}$ can thus be rephrased in terms of horizontal scale, more accessible to observations, as $l(t) \sim(\rho \sigma)^{-1}$ where $\rho=\lambda / \gamma$ is the aspect ratio of the tracer fluctuations.

\subsection{Implications for the stratosphere}

In the stratospheric context, the typical vertical scale $\sigma$ at which the intermittency of the mixing is important for tracer evolution (and hence for which diffusion is not a realistic parameterization) is seen from (2.3) to be of the order of $50 \mathrm{~m}$. As in the idealised model of the previous section, this vertical scale can be associated to a horizontal scale: as discussed by Haynes \& Anglade (1997), the generation of fine tracer structure by realistic stratospheric winds leads to a typical ratio $\rho$ of the horizontal to the vertical scales, which they found to be about 250. This estimate suggests that non-diffusive effects should be taken into account when modelling the impact of small-scale mixing on tracers with horizontal scales of the order

of $\rho \sigma \approx 15 \mathrm{~km}$ or smaller. This can be compared with the diffusive scale $\left[\kappa\left(1+\rho^{2}\right) / \gamma\right]^{1 / 2}$ obtained when balancing the effect of a large-scale strain $\gamma$ with that of small-scale mixing parameterized by diffusion (Haynes \& Anglade, 1997). This scale provides an estimate of the horizontal scale at which tracer structures, e.g. filaments, are first affected by diffusion; for $\kappa=10^{-2} \mathrm{~m}^{2} \mathrm{~s}^{-1}$, it has been found to be also of the order of $15 \mathrm{~km}$ (Haynes \& Anglade, 1997; Sparling \& Bacmeister, 2001). This is an indication of the genuine importance of the non-diffusive effects associated with mixing intermittency for stratospheric tracers: the 
horizontal scale at which these effects become significant is precisely that at which smallscale mixing itself starts to affect tracer distributions. The consequences for the statistics of stratospheric tracers, specifically for their concentration spectrum, are quantified in the next section.

\section{Stratospheric tracer spectra}

The power spectrum of the tracer concentration, that is, the Fourier transform

$$
F(\mathbf{k})=\int \mathrm{e}^{\mathrm{ik} \cdot \mathbf{r}}\langle P(\mathbf{x}+\mathbf{r}) P(\mathbf{x})\rangle \mathrm{d} \mathbf{r}
$$

of the concentration autocorrelation, provides a useful diagnostic of the statistical properties of a tracer distribution. In writing (4.1), we have made an assumption of spatial homogeneity, as is relevant to well stirred regions of the atmosphere such as the stratospheric surf zone. We make the further assumption that the tracer distribution is isotropic in the horizontal, and we concentrate on the horizontal power spectrum, that is, the power spectrum at a fixed altitude, regarded as a function of the horizontal wavenumber $k=\left|\mathbf{k}_{\mathrm{h}}\right|$.

Our objective is to compute this spectrum $F(k)$ for a tracer that is advected by observed stratospheric winds, and mixed at small scales by turbulent patches. We consider a forced-dissipative regime in which the tracer is forced at large scales, and dissipated at small scales by the random-walk parameterization of section 2 . The spectrum can then be derived by first solving a forced version of the master equation (2.7) numerically in three spatial dimensions. For a realistic strength of the mixing process, however, the use of standard numerical methods would be prohibitively expensive. We therefore employ the computationally efficient alternative provided by the WKB technique of Antonsen et al. (1996). Haynes \& Vanneste (2004) have recently used this approach to compute stratospheric tracer spec- 
tra using diffusion to parameterize small-scale mixing. The replacement of diffusion by the more complicated parameterization of section 2 changes little to the implementation of the WKB technique, which we therefore describe only briefly, referring the reader to Haynes \& Vanneste (2004) for details.

\subsection{WKB technique}

The WKB technique applies to the Batchelor regime of tracer advection, when there is a clear separation between the (large) scale of the advecting velocity field, and the (small) scale of tracer fluctuations, as is arguably the case in the lower stratosphere (see, e.g., Bartello, 2000; Shepherd et al., 2000). It is best formulated in terms of the Wigner function

$$
\omega(\mathbf{x}, \mathbf{k}, t)=\frac{1}{(2 \pi)^{3}} \int \mathrm{e}^{\mathrm{i} \mathbf{k} \cdot \mathbf{y}} P(\mathbf{x}+\epsilon \mathbf{y} / 2, t) P(\mathbf{x}-\epsilon \mathbf{y} / 2, t) \mathrm{d} \mathbf{y}
$$

which can be interpreted as a local tracer spectrum. Here, $\epsilon \ll 1$ is the relevant scaleseparation parameter, such that $\|\nabla \mathbf{v}\| /\|\nabla P\|=O(\epsilon)$.

Writing the operator $d$ in terms of the large-scale spatial variable $\mathbf{x}$ as $d\left(-\mathrm{i} \epsilon \partial_{z}\right),(2.7)$ leads to the evolution equation

$$
\begin{gathered}
\partial_{t} \omega+\frac{1}{2} \nabla \cdot \int \mathrm{e}^{\mathrm{i} \mathbf{l} \cdot \mathbf{x}} \hat{\mathbf{v}}(\mathbf{l}, t)[\omega(\mathbf{x}, \mathbf{k}+\epsilon \mathbf{l} / 2, t)+\omega(\mathbf{x}, \mathbf{k}-\epsilon \mathbf{l} / 2, t)] \mathrm{d} \mathbf{l} \\
-\frac{\mathrm{i}}{\epsilon} \mathbf{k} \cdot \int \mathrm{e}^{\mathrm{i} \mathbf{l} \cdot \mathbf{x}} \hat{\mathbf{v}}(\mathbf{l}, t)[\omega(\mathbf{x}, \mathbf{k}+\epsilon \mathbf{l} / 2, t)-\omega(\mathbf{x}, \mathbf{k}-\epsilon \mathbf{l} / 2, t)] \mathrm{d} \mathbf{l} \\
=-\left[d\left(m-\mathrm{i} \frac{\epsilon}{2} \partial_{z}\right)+d\left(m+\mathrm{i} \frac{\epsilon}{2} \partial_{z}\right)\right] \omega,
\end{gathered}
$$

for the Wigner function, where $\hat{\mathbf{v}}$ denotes the spatial Fourier transform of $\mathbf{v}$. At leading order in $\epsilon$, this equation reduces to

$$
\partial_{t} \omega+\mathbf{v} \cdot \nabla \omega-\nabla_{\mathbf{k}} \omega \cdot \nabla \mathbf{v} \cdot \mathbf{k}=-2 d(m) \omega
$$


which can be solved by integration along characteristics, according to

$$
\frac{\mathrm{d} \mathbf{x}}{\mathrm{d} t}=\mathbf{v}, \quad \frac{\mathrm{d} \mathbf{k}}{\mathrm{d} t}=-\nabla \mathbf{v} \cdot \mathbf{k}, \quad \frac{\mathrm{d} \omega}{\mathrm{d} t}=-2 d(m) \omega
$$

These ordinary differential equations describe the evolution of a fluid parcel carrying a tracer with wavevector $\mathbf{k}$ and local variance $\omega$. A tracer distribution is represented by an ensemble of such fluid parcels, and the spectrum as a function of the wavenumber $k=\left\|\mathbf{k}_{\mathrm{h}}\right\|$ is then obtained by summing the local variance $\omega$ of all parcels with a given value of $k$. When (4.2) is solved with initial condition $\mathbf{k}=\mathbf{k}_{0}$ and $\omega=\omega_{0}$ at $t=0$, this provides the spectrum of the corresponding initial-value problem (see Fereday and Haynes 2004, manuscript submitted to Phys. Fluids, for limitations of this approach, however); a time integration then yields the spectrum for the forced problem, with $\mathbf{k}_{0}$ and $\omega_{0}$ to be interpreted as the forcing wavevector and variance pumping rate, respectively. For sufficiently long integration times, this spectrum tends to a stationary form reflecting the statistical equilibrium of the forced--dissipative regime (see Haynes \& Vanneste, 2004, for details)

\subsection{Results}

We report here results obtained with the same observed stratospheric winds as in Haynes \& Vanneste (2004), that is, two-dimensional winds derived from SSU geopotential data on the 50-mb isobaric surface for January 1987. The vertical shear needed to evolve the vertical component $m$ of $\mathbf{k}$ is calculated by finite-differencing the wind on the $100-\mathrm{mb}$ and $20-\mathrm{mb}$ surfaces, and the fluid parcels are initialized to sample the Northern hemisphere surf zone. The forcing wavevectors are horizontal, with uniformly distributed azimuthal angle and $k=10^{-6} \mathrm{~m}^{-1}$.

Figure 6 compares the spectrum $F(k)$ obtained with diffusion and with the random- 
walk model of small-scale mixing, with either Gaussian or exponential step pdfs. The step variance $\sigma^{2}=2 \cdot 10^{3} \mathrm{~m}^{2}$ and inverse average waiting time $\alpha=10^{-5} \mathrm{~s}^{-1}$ have been chosen consistent with the estimates (2.3) of Alisse et al. (2000) and so as to lead to a diffusivity $\kappa=\alpha \sigma^{2} / 2$ equal to $10^{-2} \mathrm{~m}^{2} \mathrm{~s}^{-1}$. This is the value suggested by recent calculations based on high-resolution tracer data (Waugh et al., 1997; Balluch \& Haynes, 1997); it is taken here for the computation of the reference spectrum using diffusion. (The diffusive computation differs from the one reported in Haynes \& Vanneste (2004) by the fact that it does not include diffusion in the horizontal; this does not affect the form of the spectrum over the range of scales considered here.)

The effect of the intermittency of the small-scale mixing as parameterized by the randomwalk model is clearly to shallow the spectrum at small scales, as the simple models of section 3 suggest. The scale at which there is a significant departure of the random-walk spectra from the diffusive one is well predicted by the estimate $\rho \sigma$, here equal to about $11 \mathrm{~km}$, discussed in section 3.3. There is very little difference between the spectra computed with Gaussian and exponential step pdfs; this insensitivity is important since it suggests that the random-walk parameterization can be useful even in the absence of accurate estimates for $p(l)$.

A comparison of the spectra computed here with those inferred from observations of long-lived stratospheric tracers can be attempted. Bacmeister et al. (1996) and Sparling \& Bacmeister (2001) derived spectra from high-resolution aircraft measurements of $\mathrm{N}_{2} \mathrm{O}$ and $\mathrm{O}_{3}$ in the northern winter stratosphere. These spectra exhibit a relatively clear $k^{-2}$ power law over a wide range of scales, say from 50 to $500 \mathrm{~km}$. In this range, where the intermittency of the small-scale mixing plays no role, the conclusions of Haynes \& Vanneste (2004) can be repeated: because of the large variability introduced by the vertical shear, the effect of 
dissipation (be it diffusive or intermittent) extends to scales much larger than the simple estimate $\left[\kappa\left(1+\rho^{2}\right) / \gamma\right]^{1 / 2} \approx 13 \mathrm{~km}$ (here $\gamma$ is a typical horizontal strain), and the computed spectrum is roughly consistent with a $k^{-2}$ power law. For the largest scales, it tends to the $k^{-1}$ Batchelor spectrum expected on theoretical grounds; these scales, however, are not well separated from the velocity scales, so that the WKB approach is unreliable. For scales below $50 \mathrm{~km}$, there is some uncertainty in the spectra deduced from observations: using raw data sampled along aircraft tracks, Bacmeister et al. (1996) found that the $k^{-2}$ power law extends down to scales as small as $1 \mathrm{~km}$; in contrast, using a conditioning technique to remove artifacts associated with cross-isentropic sampling, Sparling \& Bacmeister (2001) obtained a spectrum that steepens at small scales, with an approximate $k^{-2.5}$ behaviour. There are also indications of considerable year-to-year variations in this part of the spectrum (Sparling \& Bacmeister, 2001). In view of this uncertainty, it is difficult to decide whether the randomwalk model leads to a more realistic spectrum than diffusion. Certainly, the random-walk model gives a spectrum that is remarkably similar to that obtained by Bacmeister et al. (1996), but it is significantly shallower at small scales than the arguably more accurate spectrum of Sparling \& Bacmeister (2001).

The spectra of Figure 6 have been obtained with what we regard as realistic values of the parameters $\sigma$ and $\alpha$. It is nevertheless interesting to examine the shape of the spectrum for other values. Figure 7 compares the spectra computed using the random-walk model with Gaussian step pdf and three distinct combinations of values for $\sigma$ and $\alpha$. These combinations have been chosen so as to have the same associated diffusivity $\kappa=\alpha \sigma^{2} / 2=10^{-2} \mathrm{~m}^{2}$ $\mathrm{s}^{-1}$. Thus, the three spectra illustrate the effect of the intermittency of small-scale mixing, with the largest value of $\sigma$ (and hence the smallest value of $\alpha$ ) corresponding to the most intermittent mixing. Increasing the intermittency clearly leads to a shallower spectrum, with 
a departure from the diffusive behaviour shifted to larger scales.

\section{Discussion}

The model of stratospheric small-scale mixing described in this paper assumes that fluid particles undergo a random walk in the vertical as a result of successive encounters with patches of three-dimensional turbulence. Because of the finiteness of both the time intervals between these encounters and of the vertical displacements, this model leads to predictions for the behaviour of tracers that differ qualitatively from those obtained with a diffusive parameterization (which assumes infinitesimal time intervals and vertical displacements). The differences emerge only for relatively short vertical scales, but with the large aspect ratio of stratospheric tracer structures, these translate into significant horizontal scales, which we estimate to be of the order of $15 \mathrm{~km}$. Coincidentally, this is of the same order as the dissipative scale, that is, the scale at which small-scale mixing needs to be taken into account. The conclusion, therefore, is this: when small-scale mixing matters for tracer evolution, it is poorly represented by diffusion.

The main non-diffusive effect that is captured by our random-walk model is the reduced efficiency of small-scale mixing in damping small-scale tracer fluctuations. Specifically, the damping rate, unlike that of diffusion, becomes scale independent in the limit of small scale, and is then given by the inverse average waiting time $\alpha$. This property has a simple physical interpretation - the sparsity of the random patches ultimately limits the efficiency of small-scale mixing - and it finds some observational support in the work of Balluch \& Haynes (1997). These authors estimated a lower-stratospheric vertical diffusivity from high-resolution aircraft tracer data by comparing the observed structure of tracer filaments 
with that predicted by a simple advection-diffusion model. Interestingly, their estimate of the diffusivity decreases strongly with the scale of the tracer filaments considered. This is consistent with the idea that the damping of tracer fluctuations is not diffusive but of a different type whose efficiency decreases with tracer scale. It would be interesting the reexamine the analysis of Balluch \& Haynes (1997) using our random-walk model rather than diffusion as a parameterization of small-scale mixing. This would provide an assessement of the validity of the random-walk model and, perhaps, a method for the estimation of the parameters $\alpha$ and $\sigma$ independent of that used in Appendix A. Other approaches for the estimation of a lower-stratospheric diffusivity (e.g. Waugh et al., 1997; Joseph et al., 2003) could be similarly re-examined.

The parameterization of small-scale mixing provided by the random-walk model has the advantage of relative simplicity: the operator it involves is a simple convolution (which may be computed efficiently using a fast Fourier transform) and, in its simplest form, is defined by only two parameters, $\alpha$ and $\sigma$. In this paper, these are estimated using the balloon data of Alisse \& Sidi (2000) and assumptions about the lifetime of turbulent patches. The limitations of the dataset used (its lack of representative spatial and temporal coverage in particular), and the fragility of the assumptions on patch lifetime mean that our estimates have to be taken with caution. It would be highly desirable to complement them by others derived from different observations of stratospheric turbulence. Of particular interest here would be data providing temporal information about the turbulent patches, allowing for a reliable estimation of the patch-lifetime and waiting-time statistics. Laboratory experiments and computer simulations could prove valuable in this respect. Ultimately, one would hope to estimate not only $\alpha$ and $\sigma$, but the full waiting-time and displacement pdfs $\psi(\tau)$ and $p(l)$ so as to avoid the ad hoc assumptions of Poisson and Gaussian (or exponential) distributions 
made in this paper.

Acknowledgements. This work stems from collaborations with J.-R. Alisse and P. H. Haynes; it is a pleasure to acknowledge their contributions and that of E. Hernández-Garcia who provided a derivation of the master equation (2.6). JV is funded by a NERC Advanced Fellowship.

\section{A Relating $\sigma$ and $\alpha$ to turbulent-patch properties}

The random-walk model of mixing of section 2 is defined by two pdfs, the pdf of vertical displacements $p(l)$, and the pdf of waiting times $\psi(\tau)$. Ultimately, these two pdfs are determined by the dynamics of small-scale turbulence in the stratosphere on the one hand, and by the sampling of this turbulence by Lagrangian trajectories. In this Appendix, we show how they can also be related to properties of turbulent patches which may be observed directly, e.g., using instrumented balloons. To this end, we refine a model due to Dewan (1981) which assumes that patches form at random times and at random altitudes. Our refinement relaxes the hypothesis made by Dewan (1981) of perfect mixing, that is, of complete homogenization of tracer concentration inside each patch. Observations suggest in fact that irreversible effects within turbulent patches are generally too weak and patch lifetimes too short for complete homogenization to take place (Alisse \& Sidi, 2000).

The model which we now describe has previously been used by Alisse et al. (2000) for the estimation of an average vertical diffusivity in the stratosphere. It is based on the characterization of turbulent patches by a set of 3 parameters, $\mathbf{p}=\left(h_{\mathrm{p}}, \kappa_{\mathrm{p}}, \tau_{\mathrm{p}}\right)$, where $h_{\mathrm{p}}$ is the height of the patch, $\kappa_{\mathrm{p}}$ its (constant) diffusivity, and $\tau_{\mathrm{p}}$ its lifetime. The mixing inside turbulent patches is described by a diffusion process which lasts during the patch 
lifetime. Such a model is of course very crude, in particular because the characteristics of turbulent patches evolve during their life cycle; but, in the absence of detailed information on this evolution, the simplest assumptions are made. A further motivation for this model is provided by experimental and observational work in which microstructure measurements are employed to infer a local diffusivity $\kappa_{\mathrm{l}}$ through the formula $\kappa_{\mathrm{l}}=\epsilon_{\mathrm{p}} / N^{2}$, where $\epsilon_{\mathrm{p}}$ is the dissipation rate of available potential energy and $N^{2}$ the Brunt-Väisälä frequency. The local diffusivity $\kappa_{1}$, which is non-negligible only inside turbulent patches, is naturally identified with the patch diffusivity $\kappa_{\mathrm{p}}$.

In the model, the statistics of turbulent patches are defined by a function $\phi(\mathbf{p})$ giving the joint pdf for a patch to have a height, diffusivity and lifetime given by $\mathbf{p}=\left(h_{\mathrm{p}}, \kappa_{\mathrm{p}}, \tau_{\mathrm{p}}\right)$. The pdf of vertical displacements can be derived from $\phi(\mathbf{p})$ according to

$$
p(l)=\frac{1}{\bar{h}_{\mathrm{p}}} \int h_{\mathrm{p}} p(l \mid \mathbf{p}) \phi(\mathbf{p}) \mathrm{d} \mathbf{p},
$$

where

$$
\int \cdot \mathrm{d} \mathbf{p}=\int_{0}^{\infty} \int_{0}^{\infty} \int_{0}^{\infty} \cdot \mathrm{d} h_{\mathrm{p}} \mathrm{d} \kappa_{\mathrm{p}} \mathrm{d} \tau_{\mathrm{p}}
$$

$p(l \mid \mathbf{p})$ is the probability for a particle to experience a displacement $l$, conditioned to its presence in a patch with characteristics $\mathbf{p}$, and the overbar denotes average over patch statistics, that is,

$$
\bar{h}_{\mathrm{p}}=\int h_{\mathrm{p}} \phi(\mathbf{p}) \mathrm{d} \mathbf{p}
$$

Let us compute $p(l \mid \mathbf{p})$. We start by assuming that the particle is initially at a distance $y$ from the centre of the patch. Its position $z$ at the end of the life time $\tau_{\mathrm{p}}$ of the patch is found by considering a Brownian motion with reflecting boundaries at $\pm h_{\mathrm{p}} / 2$. The pdf of $z$ is then given by the series (Gardiner, 1985, p. 133)

$$
Q(z \mid y)=\frac{1}{h_{\mathrm{p}}}+\frac{2}{h_{\mathrm{p}}} \sum_{n=1}^{\infty} \cos \left[\frac{n \pi\left(z+h_{\mathrm{p}} / 2\right)}{h_{\mathrm{p}}}\right] \cos \left[\frac{n \pi\left(y+h_{\mathrm{p}} / 2\right)}{h_{\mathrm{p}}}\right] \mathrm{e}^{-n^{2} \delta_{\mathrm{p}}} .
$$


In this expression, $\delta_{\mathrm{p}}=\pi^{2} \kappa_{\mathrm{p}} \tau_{\mathrm{p}} / h_{\mathrm{p}}^{2}$ characterizes the degree of mixing of the patch with characteristics $\mathbf{p}$; it can be interpreted as the ratio between the variance of the particle position at the end of the patch lifetime and the square of the patch height. The probability $p(l \mid \mathbf{p})$ is then calculated by noting that $l=z-y\left(\right.$ with the condition $\left.l \in\left[-h_{\mathrm{p}} / 2-y, h_{\mathrm{p}} / 2-y\right]\right)$ and that $y$ is uniformly distributed in $\left[-h_{\mathrm{p}} / 2, h_{\mathrm{p}} / 2\right]$ (cf. Figure 1 in Vanneste \& Haynes (2000)). Thus, for $0 \leq l \leq h_{\mathrm{p}}$,

$$
\begin{aligned}
p(l \mid \mathbf{p}) & =\frac{1}{h_{\mathrm{p}}} \int_{-h_{\mathrm{p}} / 2}^{h_{\mathrm{p}} / 2-l} Q(l+y \mid y) \mathrm{d} y \\
& =\frac{h_{\mathrm{p}}-l}{h_{\mathrm{p}}^{2}}+\frac{1}{h_{\mathrm{p}}^{2}} \sum_{n=0}^{\infty}\left[\left(h_{\mathrm{p}}-l\right) \cos \left(\frac{n \pi l}{h_{\mathrm{p}}}\right)-\frac{h_{\mathrm{p}}}{n \pi} \sin \left(\frac{n \pi l}{h_{\mathrm{p}}}\right)\right] \mathrm{e}^{-n^{2} \delta_{\mathrm{p}}} .
\end{aligned}
$$

For $-h_{\mathrm{p}} \leq l \leq 0, p(l \mid \mathbf{p})$ is obtained by symmetry; for $l \notin\left[-h_{\mathrm{p}}, h_{\mathrm{p}}\right]$ it vanishes.

It is instructive to examine the dependence of $p(l \mid \mathbf{p})$ on the patch characteristics. Figure 8 use normalized variables and displays $h_{\mathrm{p}} p(l \mid \mathbf{p})$ as a function of $l / h_{\mathrm{p}}$ for different values of $\delta_{\mathrm{p}}$. For $\delta_{\mathrm{p}} \gg 1$, illustrated here by $\delta_{\mathrm{p}}=10$, the straight line $h_{\mathrm{p}} p(l \mid \mathbf{p})=1-l / h_{\mathrm{p}}$ corresponding to perfect mixing is obtained (cf. Vanneste \& Haynes, 2000): in this case, the particle displacements are only limited by the height of the patch. For finite values of $\delta_{\mathrm{p}}$, the displacements are in addition limited by the finite diffusivity and finite lifetime of the patch. Note that even for small $\delta_{\mathrm{p}}$ the finite extent of the patch is significant because it constrains the motion of particles located near the patch boundaries.

From $p(l \mid \mathbf{p})$, the variance $\sigma_{\mathrm{p}}^{2}$ of the displacements inside a patch of given characteristics is readily found to be

$$
\sigma_{\mathrm{p}}^{2}=\int_{-\infty}^{\infty} l^{2} p(l \mid \mathbf{p}) \mathrm{d} l=\frac{h_{\mathrm{p}}^{2}}{6}\left[1-\sum_{n=0}^{\infty} b_{n} \mathrm{e}^{-(2 n+1)^{2} \delta_{\mathrm{p}}}\right]
$$

where $b_{n}=96 /\left[(2 n+1)^{4} \pi^{4}\right]$. The variance $\sigma^{2}$ associated with an ensemble of patches is then 
deduced from the equalities

$$
\sigma^{2}=\int_{-\infty}^{\infty} l^{2} p(l) \mathrm{d} l=\frac{1}{\bar{h}_{\mathrm{p}}} \int_{-\infty}^{\infty} \int l^{2} h_{\mathrm{p}} p(l \mid \mathbf{p}) \phi(\mathbf{p}) \mathrm{d} l \mathrm{~d} \mathbf{p}=\frac{1}{\bar{h}_{\mathrm{p}}} \int h_{\mathrm{p}} \sigma_{\mathrm{p}}^{2} \phi(\mathbf{p}) \mathrm{d} \mathbf{p} .
$$

It is conveniently written as

$$
\sigma^{2}=\frac{\overline{h_{\mathrm{p}}^{3}}}{6 \bar{h}_{\mathrm{p}}}(1-c)
$$

where the parameter $c$, defined by

$$
c=\frac{1}{\overline{h_{\mathrm{p}}^{3}}} \sum_{n=0}^{\infty} b_{n} \int h_{\mathrm{p}}^{3} \phi(\mathbf{p}) \mathrm{e}^{-(2 n+1)^{2} \delta_{\mathrm{p}}} \mathrm{d} \mathbf{p}
$$

and satisfying $0 \leq c \leq 1$, characterizes the imperfection of the mixing. In the limit of large typical $\delta_{\mathrm{p}}, c \rightarrow 0$ and the perfect-mixing expression $\sigma^{2}=\overline{h_{\mathrm{p}}^{3}} /\left(6 \bar{h}_{\mathrm{p}}\right)$ of Vanneste \& Haynes (2000, Appendix B) is recovered. In the opposite limit of small $\delta_{\mathrm{p}}$, corresponding to highly imperfect mixing, the property $\Sigma_{n} b_{n}=1$ can be used to show that $c \rightarrow 1$ and hence $\sigma^{2} \rightarrow 0$, corresponding to a vanishing mixing. It is interesting to derive the leading-order term of $\sigma^{2}$ in this limit. Expanding the exponentials in the definition of $c$ to first order, and using the definition of $\delta_{\mathrm{p}}$ and $b_{n}$, we find

$$
c \sim 1-\frac{1}{\overline{h_{\mathrm{p}}^{3}}} \sum_{n=0}^{\infty} \frac{96}{(2 n+1)^{2} \pi^{2}} \overline{h_{\mathrm{p}} \kappa_{\mathrm{p}} \tau_{\mathrm{p}}}
$$

Introducing this result into (A.1) and using the property $\Sigma_{n}(2 n+1)^{-2}=\pi^{2} / 8$ leads to

$$
\sigma^{2} \sim \frac{2 \overline{h_{\mathrm{p}} \kappa_{\mathrm{p}} \tau_{\mathrm{p}}}}{\bar{h}_{\mathrm{p}}}
$$

In addition to the step variance $\sigma^{2}$, the inverse average waiting time $\alpha$ is required for the random-walk parameterization of small-scale mixing. This parameter can be related to the statistics of patch lifetime and to a readily observable quantity, the fraction $F$ of the atmospheric column which is occupied by turbulent patches at any given time. This relationship is deduced by considering the number $n$ of turbulent patches that are present 
in a column of atmosphere of height $H$. Applying Little's theorem of queueing theory (e.g. Papoulis, 1991, p. 617) it can be expressed as the ratio of the average patch lifetime to $T$, the average time between the successive appearance of patches in the column:

$$
n=\frac{1}{T} \int \tau_{\mathrm{p}} \phi(\mathbf{p}) \mathrm{d} \mathbf{p}
$$

Similarly, the number $n\left(h_{\mathrm{p}}\right)$ of patches with height less that $h_{\mathrm{p}}$ is given by

$$
n\left(h_{\mathrm{p}}\right)=\frac{1}{T} \int_{0}^{h_{\mathrm{p}}} \int_{0}^{\infty} \int_{0}^{\infty} \tau_{\mathrm{p}} \phi\left(h_{\mathrm{p}}^{\prime}, \kappa_{\mathrm{p}}, \tau_{\mathrm{p}}\right) \mathrm{d} h_{\mathrm{p}}^{\prime} \mathrm{d} \kappa_{\mathrm{p}} \mathrm{d} \tau_{\mathrm{p}}
$$

$F$ is therefore given by

$$
F=\frac{1}{T H} \int_{0}^{\infty} h_{\mathrm{p}} \frac{\mathrm{d} n\left(h_{\mathrm{p}}\right)}{\mathrm{d} h_{\mathrm{p}}} \mathrm{d} h_{\mathrm{p}}=\frac{\overline{h_{\mathrm{p}} \tau_{\mathrm{p}}}}{T H} .
$$

Now, with the assumption of independence of turbulent patches, $T$ and $\alpha$ are related according to

$$
\alpha T=\frac{\bar{h}_{\mathrm{p}}}{H}
$$

Together with (A.2), this provides this expression

$$
\alpha=\frac{F \bar{h}_{\mathrm{p}}}{\overline{h_{\mathrm{p}} \tau_{\mathrm{p}}}}
$$

relating $\alpha$ to turbulent patch characteristics.

Equations (A.1) and (A.3) are the central results of this Appendix: they relate the two parameters $\sigma$ and $\alpha$ required for our parameterization of small-scale mixing to observable properties of the small-scale turbulence. Alisse et al. (2000) used these equations to estimate a lower-stratospheric diffusivity $\kappa=\alpha \sigma^{2} / 2$ from the balloon data of Alisse \& Sidi (2000). These data provide information on the joint distribution of patch height $h_{\mathrm{p}}$ and diffusivity $\kappa_{\mathrm{p}}$, but not on the distribution of patch lifetime $\tau_{\mathrm{p}}$ which is needed for both (A.1) and (A.3). Assuming simple forms for this distribution, characterized by an average $\bar{\tau}_{\mathrm{p}}$ of a few 
hours thought to be realistic (Woodman \& Rastogi, 1984; Barenblatt, 1996), Alisse et al. (2000) found the estimates $\sigma \approx 50 \mathrm{~m}$ and $\alpha \approx 10^{-5} \mathrm{~s}^{-1}$ given in (2.3), and correspondingly $\kappa \approx 10^{-2} \mathrm{~m}^{2} \mathrm{~s}^{-1}$. The consistency of the latter value with those obtained by radically different methods by Waugh et al. (1997) and Balluch \& Haynes (1997) gives some confidence in the estimates of $\sigma$ and $\alpha$, despite the many hypotheses underlying their derivation and the limited amount of data used (see Joseph et al., 2003, and references therein, however, for very different estimates of $\kappa$ ).

\section{B Derivation of the master equation}

Differentiating (2.5) with respect to $t$ gives

$$
\partial_{t} P(\mathbf{x}, t)=-\partial_{i}\left\langle v_{i}(\mathbf{x}, t) \delta(\mathbf{x}-\mathbf{x}(t))\right\rangle-\partial_{z}\langle\eta(t) \delta(\mathbf{x}-\mathbf{x}(t))\rangle
$$

after using (2.4). Since the velocity field is deterministic, the first term in the right-hand side simply is $-\nabla \cdot[\mathbf{v}(\mathbf{x}, t) P(\mathbf{x}, t)]$. To compute the second term, we use the property of shot-noise processes

$$
\langle\eta(t) \delta(\mathbf{x}-\mathbf{x}(t))\rangle=\alpha \sum_{n=1}^{\infty} \frac{1}{(n+1) !}\left\langle l^{n+1}\right\rangle\left\langle\frac{\delta^{n} \delta(\mathbf{x}-\mathbf{x}(t))}{\delta \eta(t)^{n}}\right\rangle
$$

(Hanggi, 1985; Rodriguez et al., 1985, Appendix A). Now, it is easy to derive from the solution of (2.4) in integral form that

$$
\frac{\delta x_{k}(t)}{\delta \eta}=\delta_{k, 3}
$$

the Kronecker symbol. It then follows that

$$
\left\langle\frac{\delta \delta(\mathbf{x}-\mathbf{x}(t))}{\delta \eta(t)}\right\rangle=-\partial_{z} P(\mathbf{x}, t)
$$


Introducing this result into (B.2) gives

$$
\langle\eta \delta(\mathbf{x}-\mathbf{x}(t))\rangle=\alpha \sum_{n=1}^{\infty} \frac{1}{(n+1) !}\left\langle l^{n+1}\right\rangle(-1)^{n} \partial_{z}^{n} P(\mathbf{x}, t)
$$

Equation (B.1) then becomes

$$
\partial_{t} P(\mathbf{x}, t)+\nabla \cdot[\mathbf{v} P(\mathbf{x}, t)]=\alpha \sum_{n=1}^{\infty} \frac{(-1)^{n}}{n !}\left\langle l^{n}\right\rangle \partial_{z}^{n} P(\mathbf{x}, t),
$$

after reindexing the series and assuming that the random walk is symmetric, so that $\langle l\rangle=0$. Using the definition of the average $\langle\cdot\rangle$, the right-hand side of this equation can then be rewritten

$$
\sum_{n=1}^{\infty} \frac{(-1)^{n}}{n !}\left\langle l^{n}\right\rangle \partial_{z}^{n} P(\mathbf{x}, t)=\int_{-\infty}^{\infty}\left(\mathrm{e}^{-l \partial_{z}}-1\right) P(\mathbf{x}, t) p(l) d l .
$$

Noting that $\exp \left(-l \partial_{z}\right) P(\mathbf{x}, t)=P(\mathbf{x}-l \hat{\mathbf{z}}, t)$, and changing the integration variables in (B.3) leads to the master equation in the final form (2.7). 


\section{References}

Alisse, J. \& Sidi, C. 2000, Experimental probability density functions of small-scale fluctuations in the stably stratified atmosphere, J. Fluid Mech. 402, 137-162.

Alisse, J.-R., Haynes, P. H., Vanneste, J. \& Sidi, C. 2000, Estimation of lower-stratospheric diffusivity from microstructure measurements, Geophys. Res. Lett. 27, 2621-2624.

Antonsen, T. M., Fan, Z., Ott, E. \& Garcia-Lopez, E. 1996, The role of chaotic orbits in the determination of power spectra of passive scalar, Phys. Fluids 8, 3094-3104.

Bacmeister, J. T., Eckermann, S. D., Newman, P. A., Lait, L., Chan, K. R., Loewenstein, M., Proffitt, M. H. \& Gary, B. L. 1996, Stratospheric horizontal wavenumber spectra of winds, potential temperature, and atmospheric tracers observed by high-altitude aircraft, J. Geophys. Res. 101, 9441-9470.

Balluch, M. \& Haynes, P. H. 1997, Quantification of lower stratospheric mixing processes using aircraft data, J. Geophys. Res. 102, 23487-23504.

Barenblatt, G. I. 1996, Scaling, self-similarity, and intermediate asymptotics, Cambridge University Press.

Bartello, P. 2000, Using low-resolution winds to deduce fine structure in tracers, Atmos.Ocean 38, 303-320.

Dewan, E. M. 1980, A one-dimensional vertical diffusion parameter for extremely inhomogeneous layered turbulence in stratified fluids, Technical Report AFGL-TR-80-0186, U.S. Air Force Geophysics Laboratory. 
Dewan, E. M. 1981, Turbulent vertical transport due to thin intermittent mixing layers in the stratosphere and other stable fluids, Science 211, 1041-1042.

Gardiner, C. W. 1985, Handbook of stochastic methods, 2nd edn, Springer-Verlag.

Hanggi, P. 1985, The functional derivative and its use in the description of noisy dynamical systems, in L. Pesquera \& M. A. Rodriguez, eds, Stochastic processes applied to physics, World Scientific, pp. 69-95.

Haynes, P. H. \& Anglade, J. 1997, The vertical-scale cascade in atmospheric tracers due to large-scale differential advection, J. Atmos. Sci. 54, 1121-1136.

Haynes, P. H. \& Vanneste, J. 2004, Stratospheric tracer spectra, J. Atmos. Sci. 61, 161-178.

Joseph, B., Legras, B. \& Lefèvre, F. 2003, Vertical diffusivity in the lower stratosphere from Lagrangian back-trajectory reconstructions of ozone profiles, J. Geophys. Res. D18, 4562.

Montroll, E. W. \& Weiss, G. H. 1965, Random walks on lattices. II, J. Math. Phys. 6, 167181.

Papoulis, A. 1991, Probability, random variables, and stochastic processes, 3nd edn, McGraw-Hill.

Rodriguez, M. A., Pesquera, L., San Miguel, M. \& Sancho, J. M. 1985, Master equation description of external Poisson white noise in finite systems, J. Stat. Phys. 40, 669-725.

Shepherd, T., Koshyk, J. N. \& Ngan, K. 2000, On the nature of large-scale mixing in the stratosphere and mesosphere, J. Geophys. Res. D105, 12433-12446.

Sparling, L. C. \& Bacmeister, J. T. 2001, Scale dependence of tracer microstructure: Pdfs, intermittency and the dissipation scale, Geophys. Res. Lett. 28, 2823-2826. 
Vanneste, J. \& Haynes, P. H. 2000, Intermittent mixing in strongly stratified geophysical fluids as a random walk, J. Fluid Mech. 411, 165-185.

Waugh, D. W., Plumb, R. A., Elkins, J. W., Fahey, D. W., Boering, K. A., Dutton, G. S., Keim, E., Gao, R.-S., Daube, B. C., Wofsky, S. C., Loewenstein, M., Podolske, J. R., Chan, K. R., Profitt, M. H., Kelly, K. K., Newman, P. A. \& Lait, L. R. 1997, Mixing of polar air into middle latitudes as revealed by tracer-tracer scatter plots, J. Geophys. Res. 102, 13119-13134.

Weiss, G. H. \& Rubin, R. J. 1983, Random walks: theory and selected applications, Adv. Chem. Phys. 52, 363-505.

Woodman, R. F. \& Rastogi, P. K. 1984, Evaluation of effective eddy diffusivity coefficients using radar observations of turbulence in the stratosphere, Geophys. Res. Lett. 11, 243246. 


\section{List of Figures}

1 Schematic of the vertical motion of a fluid particle in the random-walk model: the small-scale mixing inside each turbulent patch results in a net vertical displacement $l$ taken to be random. The waiting time $\tau$ between successive encounters with turbulent patches is a random variable with Poisson distri-

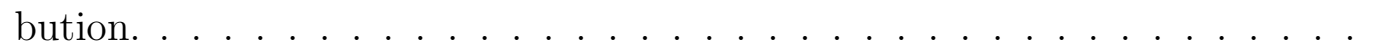

2 Dimensionless decay rate $d / \alpha$ of tracer fluctuations as a function of their dimensionless wavenumber $\sigma m$. The diffusive decay rate (diff) is compared to the decay rate obtained with the random-walk model and Gaussian (g) or exponential $($ e) step pdfs. . . . . . . . . . . . . . . . . .

3 Scaled decay factor $B$ defined by (3.2) and (3.5) as a function of the dimensionless time $\gamma t$ for a tracer in a strained flow. The diffusive result (diff) is compared with the results of the random-walk model with Gaussian (g) or exponential (e) step pdfs. . . . . . . . . . . . . . . . . . . .

4 Dimensionless slope at the origin of the equilibrium concentration profile obtained for a tracer in a strain flow. The slopes obtained with the random-walk model with Gaussian (g) and exponential (e) step pdfs are normalized by the slope obtained with diffusion and shown as a function of $\beta=\gamma /(2 \alpha)$. . .

5 Equilibrium concentration profile obtained for a tracer in a strain flow: the profile obtained for the random-walk model with $\beta=\gamma /(2 \alpha)=1$ and an exponential step pdf (e) is compared with the diffusive profile (diff). . . . . 
6 Stationary tracer spectrum as a function of the horizontal wavenumber $k$. The spectrum obtained with diffusion (diff) is compared with the spectra obtained with the random-walk model of small-scale mixing and Gaussian (g) or exponential (e) step pdfs. The step variance and inverse average waiting time are $\sigma^{2}=2 \cdot 10^{3} \mathrm{~m}^{2}$ and $\alpha=10^{-5} \mathrm{~s}^{-1}$; the corresponding diffusivity $\kappa=\alpha \sigma^{2} / 2=10^{-2} \mathrm{~m}^{2} \mathrm{~s}^{-1}$. A $k^{-2}$ power law is also shown. . . . . . . . .

7 Stationary tracer spectra as a function of the horizontal wavenumber $k$ obtained with the random-walk model of mixing and a Gaussian step pdf. The step variance and inverse average waiting times are: (a) $\sigma^{2}=8 \cdot 10^{3} \mathrm{~m}^{2}$, $\alpha=2.5 \cdot 10^{-6} \mathrm{~s}^{-1} ;$ (b) $\sigma^{2}=2 \cdot 10^{3} \mathrm{~m}^{2}, \alpha=10^{-5} \mathrm{~s}^{-1} ;$ (c) $\sigma^{2}=5 \cdot 10^{2} \mathrm{~m}^{2}$, $\alpha=4 \cdot 10^{-5} \mathrm{~s}^{-1}$. The corresponding diffusivity $\kappa=\alpha \sigma^{2} / 2=10^{-2} \mathrm{~m}^{2} \mathrm{~s}^{-1}$ is the same in all cases. The spectrum obtained with diffusion (diff) and a $k^{-2}$ power law are also shown. . . . . . . . . . . . . . .

8 Normalised pdf for the size $l$ of the vertical displacement experienced by a fluid particle in a turbulent patch. The five curves correspond to different values of the parameter $\delta_{\mathrm{p}}=\pi^{2} \kappa_{\mathrm{p}} \tau_{\mathrm{p}} / h_{\mathrm{p}}^{2}$ characterizing the patch. . . . . . 


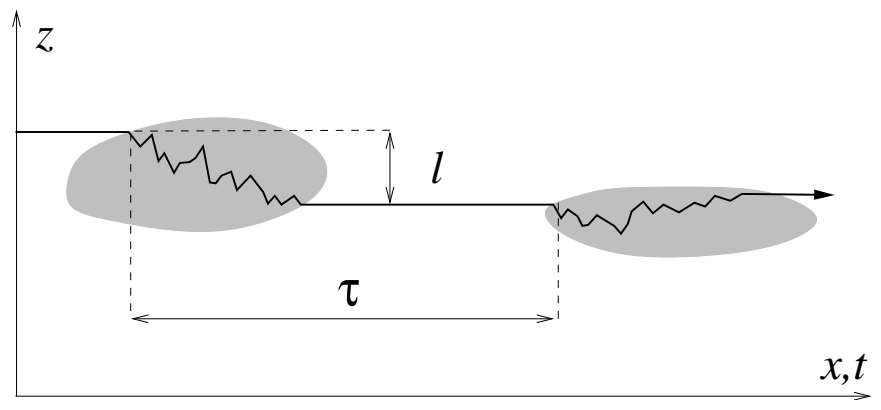

Figure 1: Schematic of the vertical motion of a fluid particle in the random-walk model: the small-scale mixing inside each turbulent patch results in a net vertical displacement $l$ taken to be random. The waiting time $\tau$ between successive encounters with turbulent patches is a random variable with Poisson distribution. 


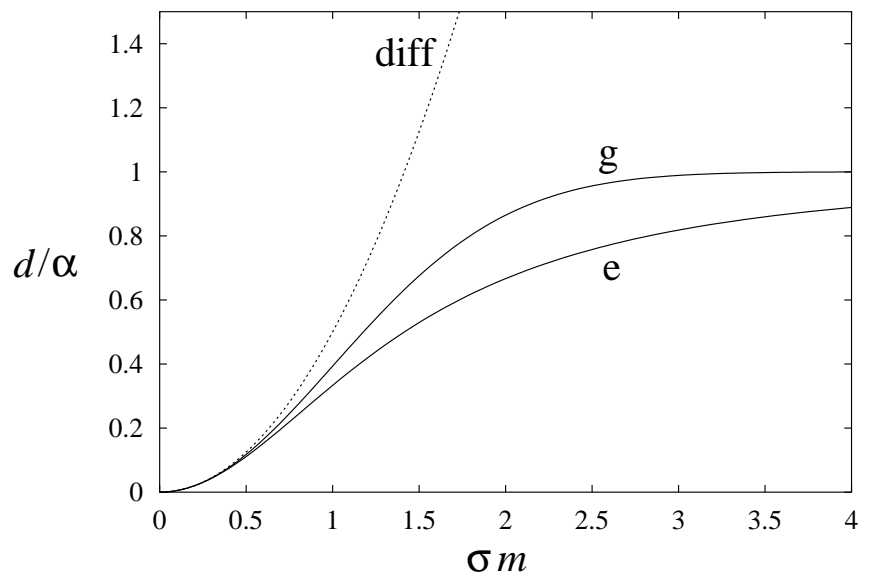

Figure 2: Dimensionless decay rate $d / \alpha$ of tracer fluctuations as a function of their dimensionless wavenumber $\sigma m$. The diffusive decay rate (diff) is compared to the decay rate obtained with the random-walk model and Gaussian (g) or exponential (e) step pdfs. 


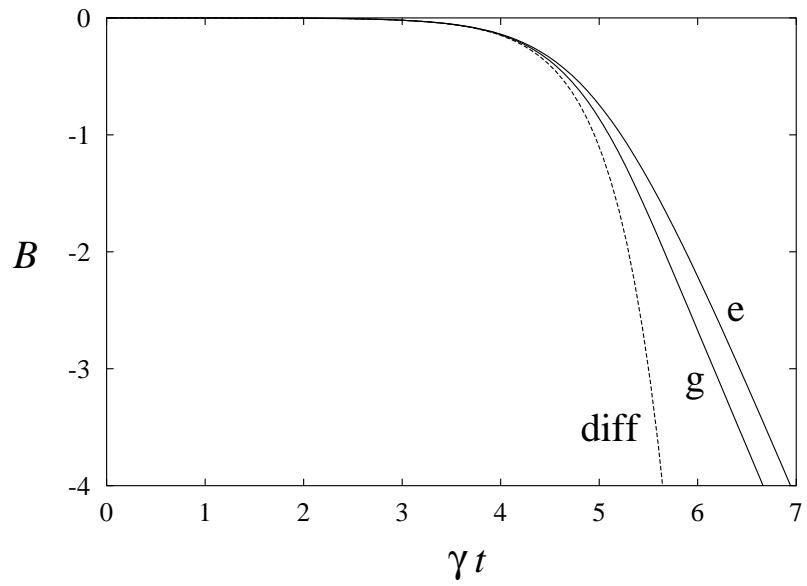

Figure 3: Scaled decay factor $B$ defined by (3.2) and (3.5) as a function of the dimensionless time $\gamma t$ for a tracer in a strained flow. The diffusive result (diff) is compared with the results of the random-walk model with Gaussian (g) or exponential (e) step pdfs. 


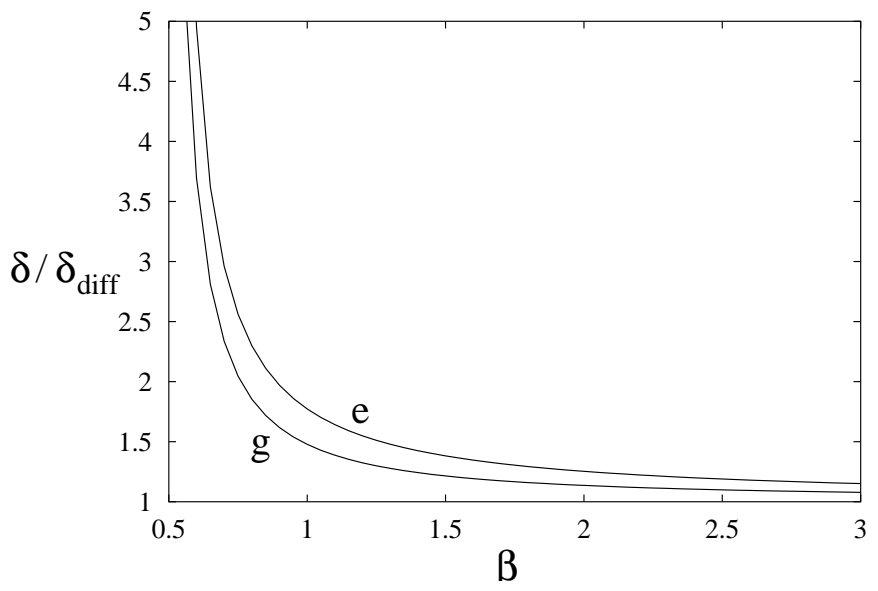

Figure 4: Dimensionless slope at the origin of the equilibrium concentration profile obtained for a tracer in a strain flow. The slopes obtained with the random-walk model with Gaussian (g) and exponential (e) step pdfs are normalized by the slope obtained with diffusion and shown as a function of $\beta=\gamma /(2 \alpha)$. 


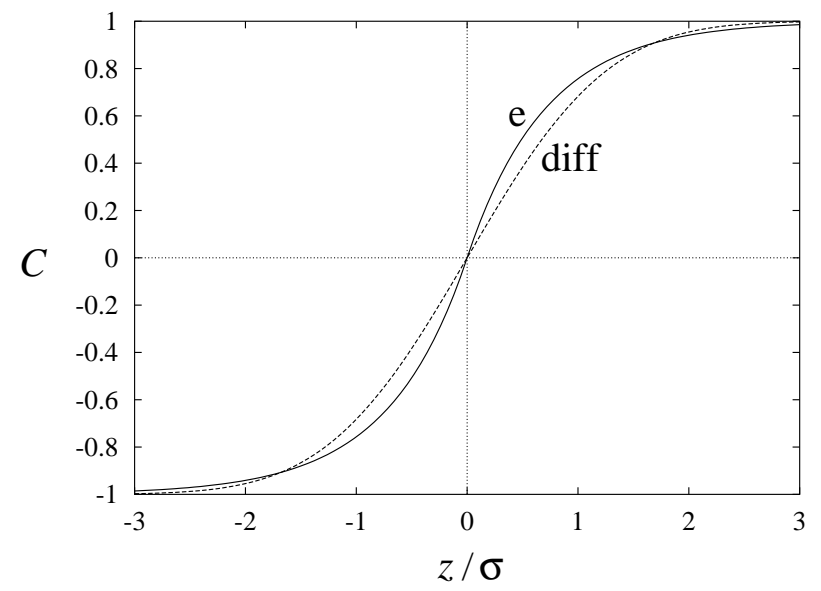

Figure 5: Equilibrium concentration profile obtained for a tracer in a strain flow: the profile obtained for the random-walk model with $\beta=\gamma /(2 \alpha)=1$ and an exponential step pdf (e) is compared with the diffusive profile (diff). 


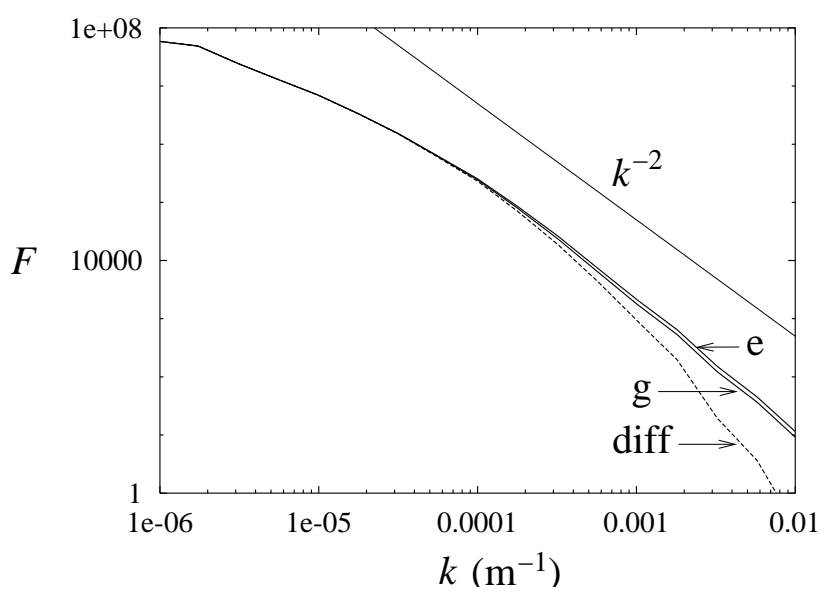

Figure 6: Stationary tracer spectrum as a function of the horizontal wavenumber $k$. The spectrum obtained with diffusion (diff) is compared with the spectra obtained with the random-walk model of small-scale mixing and Gaussian (g) or exponential (e) step pdfs. The step variance and inverse average waiting time are $\sigma^{2}=2 \cdot 10^{3} \mathrm{~m}^{2}$ and $\alpha=10^{-5} \mathrm{~s}^{-1}$; the corresponding diffusivity $\kappa=\alpha \sigma^{2} / 2=10^{-2} \mathrm{~m}^{2} \mathrm{~s}^{-1}$. A $k^{-2}$ power law is also shown. 


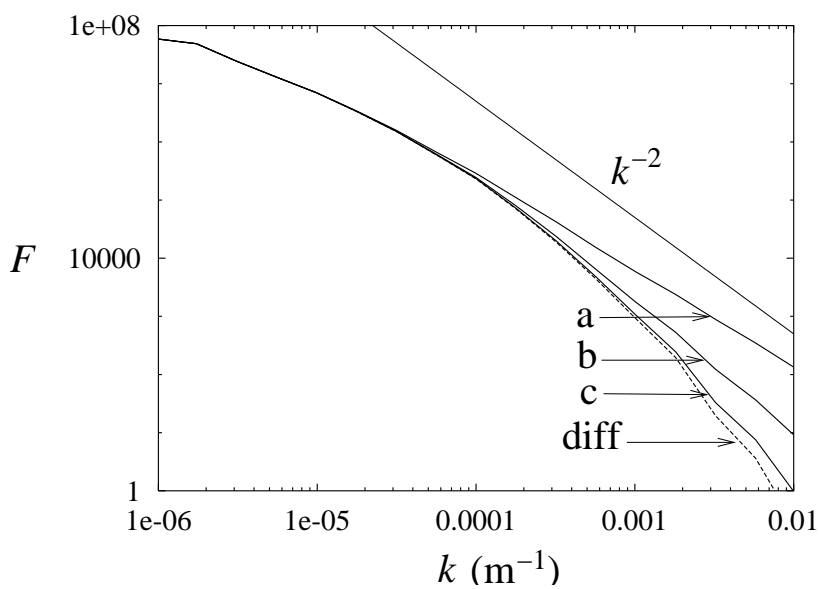

Figure 7: Stationary tracer spectra as a function of the horizontal wavenumber $k$ obtained with the random-walk model of mixing and a Gaussian step pdf. The step variance and inverse average waiting times are: (a) $\sigma^{2}=8 \cdot 10^{3} \mathrm{~m}^{2}, \alpha=2.5 \cdot 10^{-6} \mathrm{~s}^{-1}$; (b) $\sigma^{2}=2 \cdot 10^{3}$ $\mathrm{m}^{2}, \alpha=10^{-5} \mathrm{~s}^{-1} ;$ (c) $\sigma^{2}=5 \cdot 10^{2} \mathrm{~m}^{2}, \alpha=4 \cdot 10^{-5} \mathrm{~s}^{-1}$. The corresponding diffusivity $\kappa=\alpha \sigma^{2} / 2=10^{-2} \mathrm{~m}^{2} \mathrm{~s}^{-1}$ is the same in all cases. The spectrum obtained with diffusion (diff) and a $k^{-2}$ power law are also shown. 


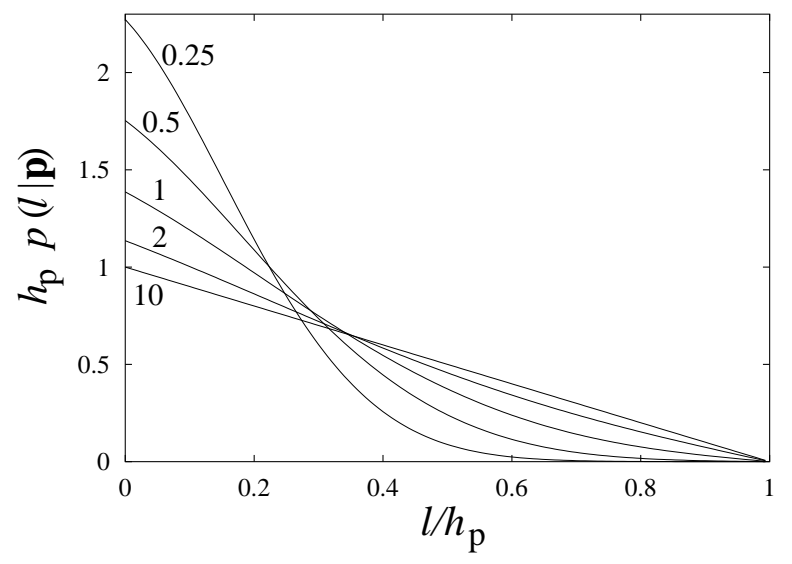

Figure 8: Normalised pdf for the size $l$ of the vertical displacement experienced by a fluid particle in a turbulent patch. The five curves correspond to different values of the parameter $\delta_{\mathrm{p}}=\pi^{2} \kappa_{\mathrm{p}} \tau_{\mathrm{p}} / h_{\mathrm{p}}^{2}$ characterizing the patch. 